\title{
Environmental Radiation Monitoring Utilizing Solid State Dosimeters
}

\author{
Shin Koyama ${ }^{1}$, Yuka Miyamoto ${ }^{1,2}$, Akihiko Fujiwara ${ }^{1}$, Haruki Kobayashi' ${ }^{1}$, \\ Katsuya Ajisawa ${ }^{1}$, Hironobu Komori ${ }^{3}$, Yoshinori Takei ${ }^{1}$, Hidehito Nanto ${ }^{1, *}$, \\ Toshio Kurobori ${ }^{4}$, Hitoshi Kakimoto ${ }^{5}$, Masaaki Sakakura ${ }^{6}$, \\ Yasuhiko Shimotsuma ${ }^{6}$, Kiyotaka Miura ${ }^{7}$, \\ Kazuyuki Hirao ${ }^{7}$ and Takayoshi Yamamoto ${ }^{2}$ \\ 'Advanced Materials Science Research and Development Center, \\ Kanazawa Institute of Technology, \\ 3-1 Yatsukaho, Hakusan, Ishikawa 924-0838, Japan \\ ${ }^{2}$ Oarai Research Center, Chiyoda Technol Corpration, \\ 3681 Narita-cho, Oarai-machi Higashi-ibaraki-gun, Ibaraki 311-1313, Japan \\ ${ }^{3}$ Nagase Landauer, C22 gaiku-1, Suwa, Tsukuba, Ibaraki 300-2686, Japan \\ ${ }^{4}$ Graduated School of Natural Science and Technology, Kanazawa University, \\ Kakuma-cho, Kanazawa, Ishikawa 920-1192, Japan \\ ${ }^{5}$ Ishikawa Prefectural Institute of Public Health and Environment Science, \\ 1-11 Taiyogaoka, Kanazawa, Ishikawa 920-1154, Japan \\ ${ }^{\circ}$ NEDO Photonics for Integrated Laboratory Kyoto University, \\ Yoshida-Honmachi, Sakyo-ku, Kyoto 606-8501, Japan \\ ${ }^{7}$ Department of Materials Chemistry, Graduate School of Engineering, Kyoto University, \\ A3-119 Katsura, Nishikyo-ku, Kyoto 615-8510, Japan
}

(Received March 29, 2010; accepted April 27, 2010)

Key words: glass dosimeter, radiophotoluminescence, environmental radiation monitoring

A personal glass dosimeter (GD) based on the radiophotoluminescence phenomenon in $\mathrm{Ag}^{+}$-doped phosphate glass was evaluated for its applicability to the measurement of environmental natural background radiation. To investigate the potential of the personal GD, we measured the monthly data of environmental natural background radiation at seven points in Ishikawa prefecture. The results indicated that the personal GD is suitable and reasonable for monitoring environmental natural background radiation. It is very important to monitor environmental natural background radiation to detect changes in environmental radiation dose such as experiments using an atomic bomb or an accidental leakage of radioactivity from a radiation facility. The personal GD will be a very useful tool to monitor both the environmental natural background radiation dose and personal radiation dose.

${ }^{*}$ Corresponding author: e-mail: hnanto@neptune.kanazawa-it.ac.jp 


\section{Introduction}

We have been exposed to radiation for as long as we have lived on the earth. It is inevitable to be exposed to environmental radiation; however, if environmental radiation did not exist, we could not have existed either. Although environmental radiation is necessary for life, the dose of the radiation governs its effect on human health. Therefore, it is important to determine the exact environmental radiation dose. So far, some types of personal dosimeter have been developed not only for personal monitoring but also for environmental radiation monitoring. For instance, a thermoluminescence dosimeter (TLD) has been studied to monitor environmental radiation. ${ }^{(1,2)}$ Recently, newly developed passive dosimeters, such as an optically stimulated luminescence (OSL) dosimeter or a direct ion storage (DIS) dosimeter have also been tried to monitor environmental radiation. ${ }^{(3)}$ Although an active dosimeter has been found to be appropriate for monitoring dose equivalent rates of environmental background radiation, passive dosimeters such as TLD, OSL and DIS have also a good potential to monitor environmental background radiation. ${ }^{(3)}$ In 2001, the Japanese law concerning radiation protection was modified and passed, that is, the dose equivalents at the boundary of the controlled area must be limited be less than or equal to $1.3 \mathrm{mSv} / 3$ months; consequently, not only dose equivalent rates but also dose equivalent can be applied to environmental radiation monitoring. In this study, we investigated the potential of a personal GD (type: GD-450, supplied by Chiyoda Technol Co., Ltd.), which is a passive dosimeter, for environmental radiation monitoring. We compared the capability of glass dosimeters with other dosimeters such as a Luxel badge based on the OSL phenomenon and a DIS dosimeter. It is found that GD-450, which is a GD, is very useful for detecting environmental radiation.

\section{Materials and Methods}

\subsection{Principle of personal dosimeters}

A personal GD of type GD-450 is made of $\mathrm{Ag}^{+}$-doped phosphate glass (AGC Techno Glass Co., Ltd.), supplied by Chiyoda Technol Corp. ${ }^{(4)}$ Another GD of type SC-1, which was developed for environmental radiation monitoring, was also used in experiments. The glass material of the SC-1 dosimeter is the same as that of GD-450; however, the filters used are different. The principle of the GD is as follows: the $\mathrm{Ag}^{+}$-doped phosphate glass after exposure to ionizing radiation exhibits an intense luminescence caused by excitation with ultraviolet light. This phenomenon is called radiophotoluminescence (RPL). When the glass is exposed to ionizing radiation, electron-hole pairs are produced. The electrons are captured into $\mathrm{Ag}^{+}$ions in the glass structure and then, the $\mathrm{Ag}^{+}$ions change to $\mathrm{Ag}^{0}$. On the other hand, the holes are captured by $\mathrm{PO}_{4}$ tetrahedrons at the beginning of the migration and then the holes produce $\mathrm{Ag}^{2+}$ ions owing to their interaction with $\mathrm{Ag}^{+}$ ions with the passing of time. It has been reported that both $\mathrm{Ag}^{0}$ and $\mathrm{Ag}^{2+}$ ions can be the center of luminescence in phosphate glass. ${ }^{(4)}$ As RPL intensity is proportional to the amount of irradiation, $\mathrm{Ag}^{+}$-doped phosphate glass can be applied to individual monitoring of ionizing radiation. Moreover, the center of luminescence will never disappear unless 
glasses are annealed at a high temperature of about $400^{\circ} \mathrm{C}$. This results in some excellent features such as repeatable measurement and small dispersions among samples.

A direct ion storage (DIS) dosimeter ${ }^{(3)}$ used for environmental background radiation monitoring in this study as one of other types of dosimeter is composed of a metaloxide-semiconductor field effect transistor (MOSFET) with an ionizing chamber. ${ }^{(5)}$ The DIS dosimeter was supplied by RADOS Technology, Finland via Nagase-Landauer Co., Ltd. The basic principle of DIS is as follows: a nonvolatile solid-state memory cell is stored in the form of an electric charge trapped on the floating gate of a MOSFET in an air or gas space surrounded by a conductive wall. The DIS dosimeter is based on an analog electrically erasable programmable read only memory (Analog-EEPROM). The DIS responds to X-, $\gamma$-, and $\beta$-rays and neutrons. This dosimeter has an excellent energy characteristic and can be read repeatably without the quenching of data. The DIS dosimeter can, therefore, detect a radiation dose within the wide range from $1 \mu \mathrm{Sv}$ to 40 Sv.

An optically stimulated luminescence (OSL) dosimeter, ${ }^{(3)}$ the so-called Luxel badge, is made of a carbon-doped $\alpha-\mathrm{Al}_{2} \mathrm{O}_{3}\left(\mathrm{Al}_{2} \mathrm{O}_{3}: \mathrm{C}\right)$ phosphor, supplied by Nagase Landauer Co., Ltd. Electrons excited by ionizing radiation are trapped by lattice defects in the phosphor, where the phenomenon is proportional to exposure dose. The phosphor emits a light (wavelength: $420 \mathrm{~nm}$ ) with an intensity proportional to the exposure dose under optical stimulation with a wavelength of $523 \mathrm{~nm}$.

\subsection{Experimental procedure}

The personal GDs such as GD-450 and SC-1 as well as DIS and Luxel badge were set at 7 points in Ishikawa prefecture, as shown in Fig. 1. Each of the seven points where the monitoring was carried out, is represented by an alphabet from A to $G$ (point A, inside of a common living house in Tsurugi-machi; point B, inside of Lower Level Radiation Laboratory in Kanazawa University at Tatunokuchi-machi; point C, inside of a house in Mt. Shishiku; point D, outside of a house in Mt. Shishiku; point E, inside of Ogoya Mines; point F, outside of Ogoya Mines; and point G, rooftop of Ishikawa Prefecture Institute of Public Health and Environmental Science) in Ishikawa prefecture. Photographs of the points where the dosimeters were set up are shown in Fig. 2. Data were obtained monthly. The accumulated monthly data were divided into daily data multiplied by 30 days. Each data point was compensated appropriately with each formula for the dosimeters. ${ }^{(3)}$ Data that were obtained at the same point were averaged and standard deviations were calculated. The data obtained using GD-450 were compared with those obtained using the other dosimeters.

\section{Results and Discussion}

Typical RPL emission and excitation spectra obtained using the X-ray-irradiated GD-450 dosimeter are shown in Fig. 3. It can be seen that the RPL emission spectrum consists of two emission bands at about $2.70 \mathrm{eV}(460 \mathrm{~nm})$ and $2.21 \mathrm{eV}(560 \mathrm{~nm})$. It can also be seen that there are two excitation bands at about $3.94 \mathrm{eV}(315 \mathrm{~nm})$ and $3.44 \mathrm{eV}$ $(360 \mathrm{~nm})$ in the near-ultraviolet wavelength region. From the viewpoint of individual 


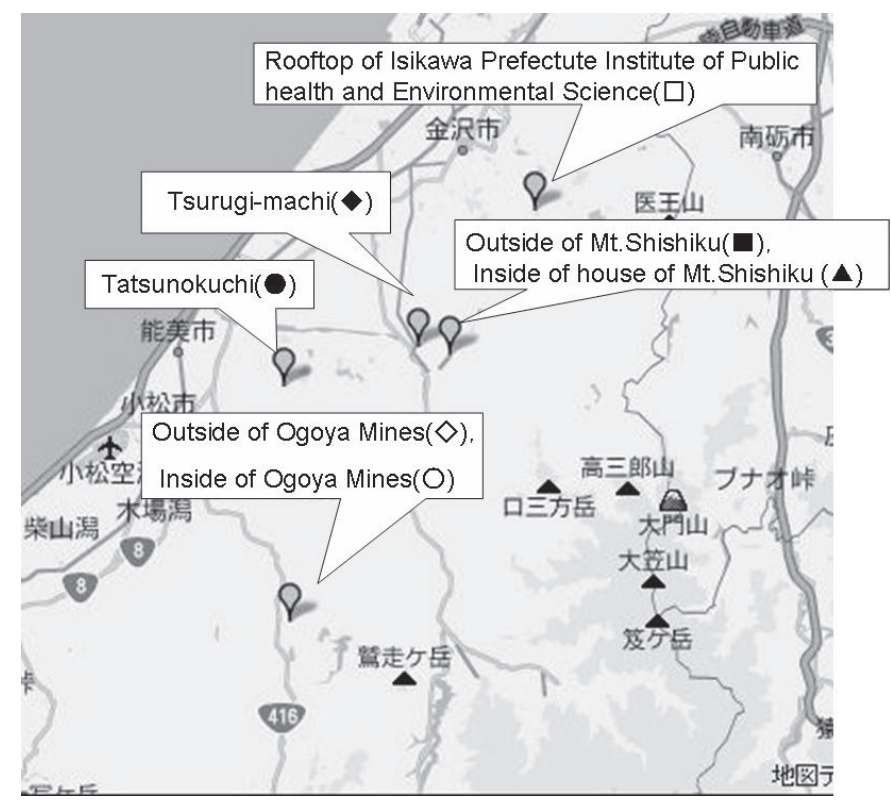

Fig. 1. Map of 7 points, namely, point A, Tsurugi-machi ( $\bullet)$; point B, inside of Lower Level Radiation Laboratory in Kanazawa University at Tatunokuchi-machi (•); point C, inside of house in Mt. Shishiku ( $\boldsymbol{\Delta})$; point D, outside of house in Mt. Shishiku (ם); point E, inside of Ogoya Mines (O); point F, outside of Ogoya Mines $(\diamond)$; and point G, rooftop of Ishikawa Prefecture Institute of Public Health and Environmental Science $(\square)$ in Ishikawa prefecture, in which environmental background radiation dose was measured using GD, DIS and Luxel badge.

and environmental monitoring of ionizing radiation, it was confirmed that RPL intensity almost linearly increased with X-ray absorption dose up to 5 Gy. ${ }^{(4)}$

Figure 4 shows photographs of the GD-450 dosimeter with and without X-ray irradiation. It can be seen that the GD-450 dosimeter, which was dark brown, exhibited an intense orange-blue fluorescence following X-ray irradiation.

Although natural background radiation doses measured with the GD-450 dosimeter at each point in Ishikawa prefecture were significantly different, the standard deviations were very small. Consequently, one can say that the GD-450 dosimeter can be suitable for monitoring environmental natural background radiation. Although the values were slightly different between GD-450 and Luxel badge, the tendencies of environmental radiation dose at each point were very similar, as shown in Fig. 5. The higher dose at point B (Tatsunokuchi) than at other points is due to the use of radioisotopes at the Lower Level Radiation Laboratory in Kanazawa University. It has been reported that the Luxel badge dosimeter based on the OSL phenomenon exhibits an excellent linearity in the dose range from $5 \times 10^{-6}$ to $50 \mathrm{~Gy}$; $^{(7)}$ the study of the mechanism of OSL in carbon-doped $\alpha-\mathrm{Al}_{2} \mathrm{O}_{3}$ phosphor is now underway. ${ }^{(8)}$ The results described above mean that GD-450 is very suitable for investigating environmental background radiation at low doses. 


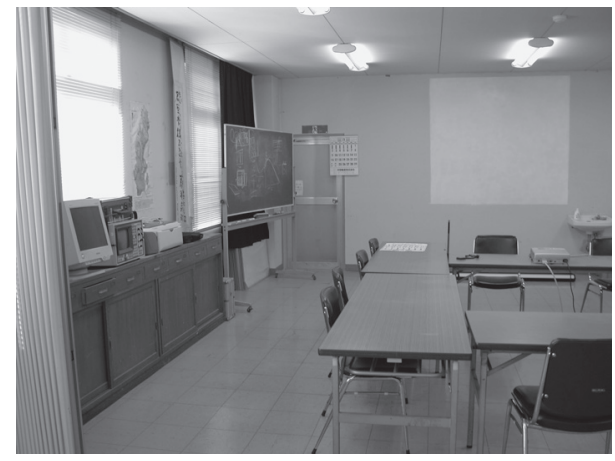

(a)

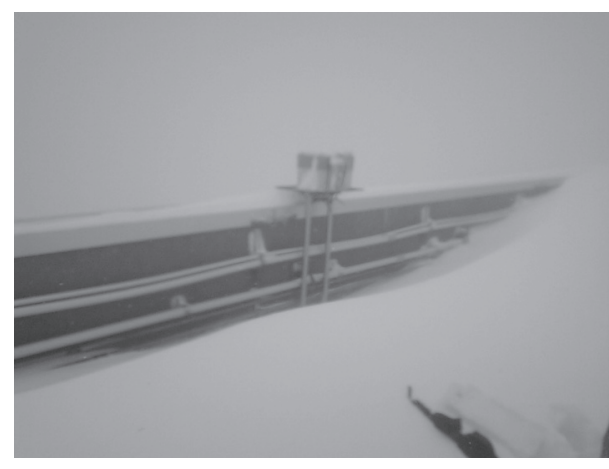

(c)

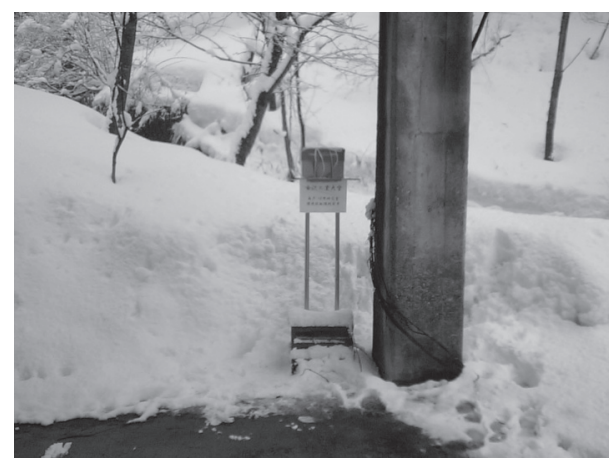

(e)

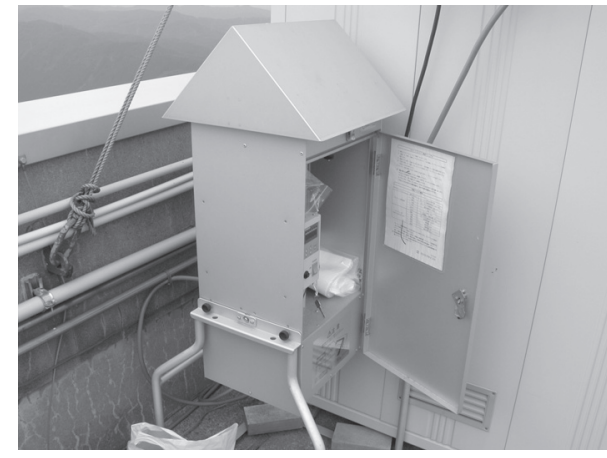

(b)

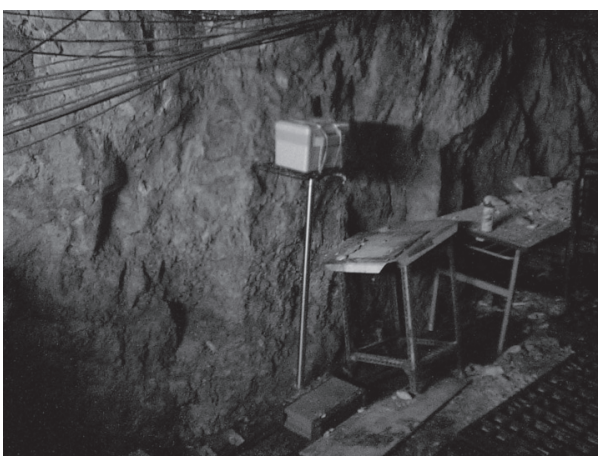

(d)

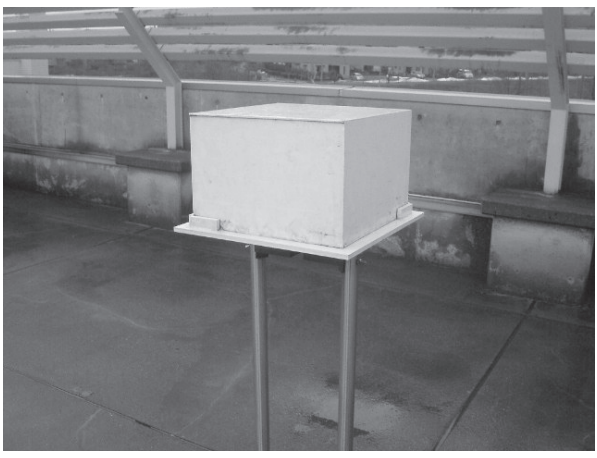

(f)

Fig. 2. Photographs of 6 points, namely, (a) point $B$, (b) point $C$, (c) point $D$, (d) point $E$, (e) point Fand (f) point $\mathrm{G}$ in Ishikawa prefecture, where the dosimeters were set up. 


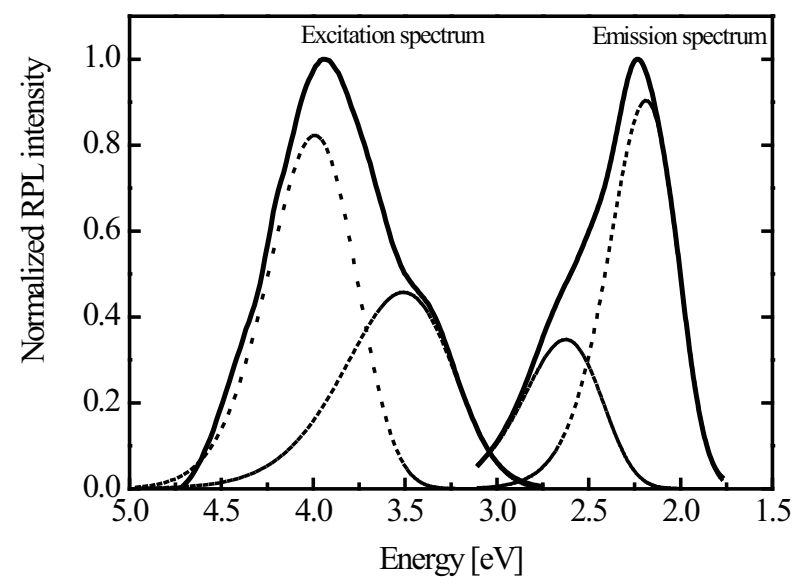

Fig. 3. Typical RPL emission and excitation spectra of $\mathrm{Ag}^{+}$-doped phosphate glass after X-ray irradiation.

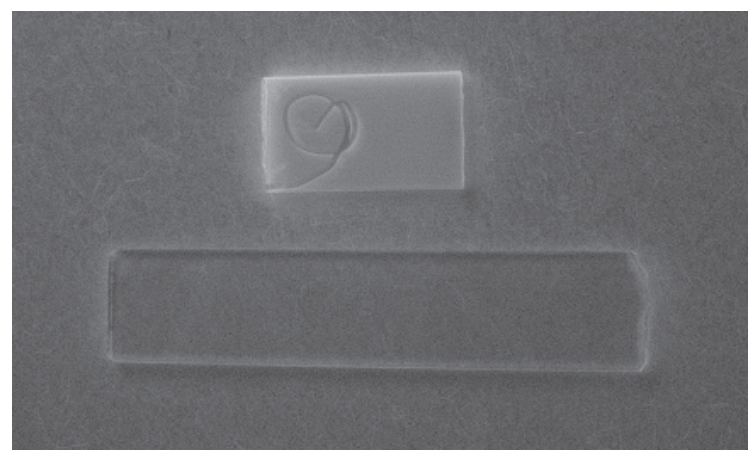

Fig. 4. Photograph of GD-450, with (upper) and without (lower) X-ray irradiation. Intense orange RPL was observed in X-ray irradiated GD.

Moreover, the values of the GD-450 and the DIS dosimeters were very close and there was no significant difference between them as shown in Fig. 6. Fuchs et al. reported new data on DIS, ${ }^{(9)}$ and they proved the adequacy of the DIS dosimeter for longterm personal dosimetry. In their study, they examined the long-term linear response behavior, calibration and readout accuracy with large samples of DIS dosimeters used, especially in the low-dose region. Vanhavere and Covens also indicated that DIS has a clear advantage in personal dosimetry. ${ }^{(10)}$ Although the DIS dosimeter has not been reported as an environmental background monitoring dosimeter up to now, it will be very useful for monitoring environmental radiation, as well as the GD-450 dosimeter. 


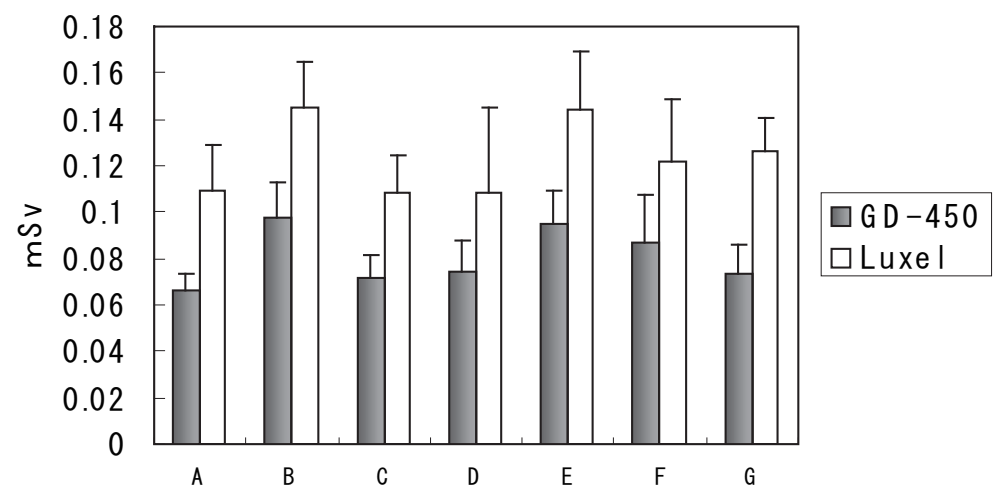

Fig. 5. Dose response at each point in Ishikawa prefecture (A: Tsurugi-machi, B: Tatsunokuchi, C: Inside of house of Mt. Shishiku, D: Outside of Mt. shishiku, E: Inside of Ogoya Mines, F: Outside of Ogoya Mines, G: Public health and Environmental Science) obtained using GD-450 (shaded bars) or Luxel badge (white bars) dosimeters.

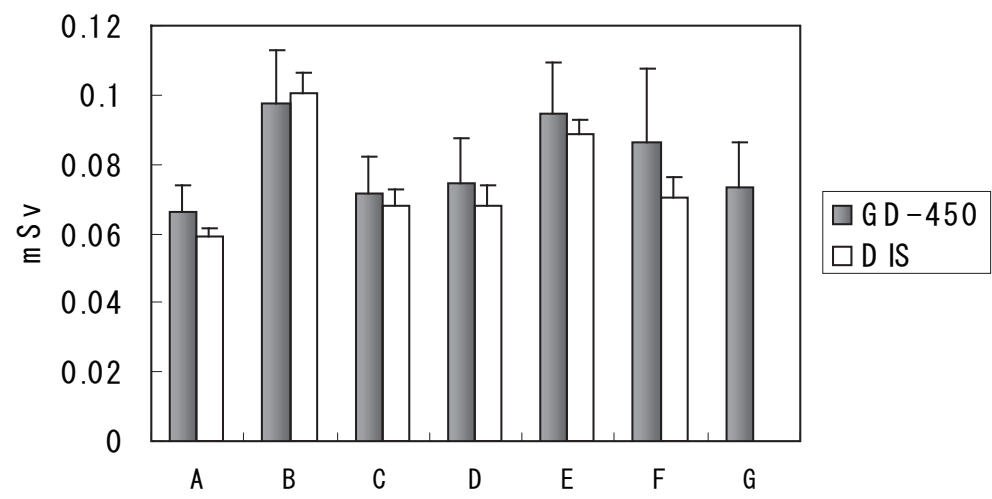

Fig. 6. Dose response at each point in Ishikawa prefecture (A: Tsurugi-machi, B: Tatsunokuchi, C: Inside of house of Mt. Shishiku, D: Outside of Mt. shishiku, E: Inside of Ogoya Mines, F: Outside of Ogoya Mines, G: Public health and Environmental Science) determined using GD-450 (shaded bars) or DIS (white bars) dosimeters. There is no data at G for DIS.

The units are not suitable for comparison between the GD-450 and SC-1 dosimeters, as the increasing and decreasing rates are very similar, as shown in Fig. 7. We carried out the ANOVA of each value at every point after adjusting the average value. Consequently, we found that there is no significant difference at each point. Monitoring natural background radiation dose with a personal GD-450 dosimeter seems to be feasible. 


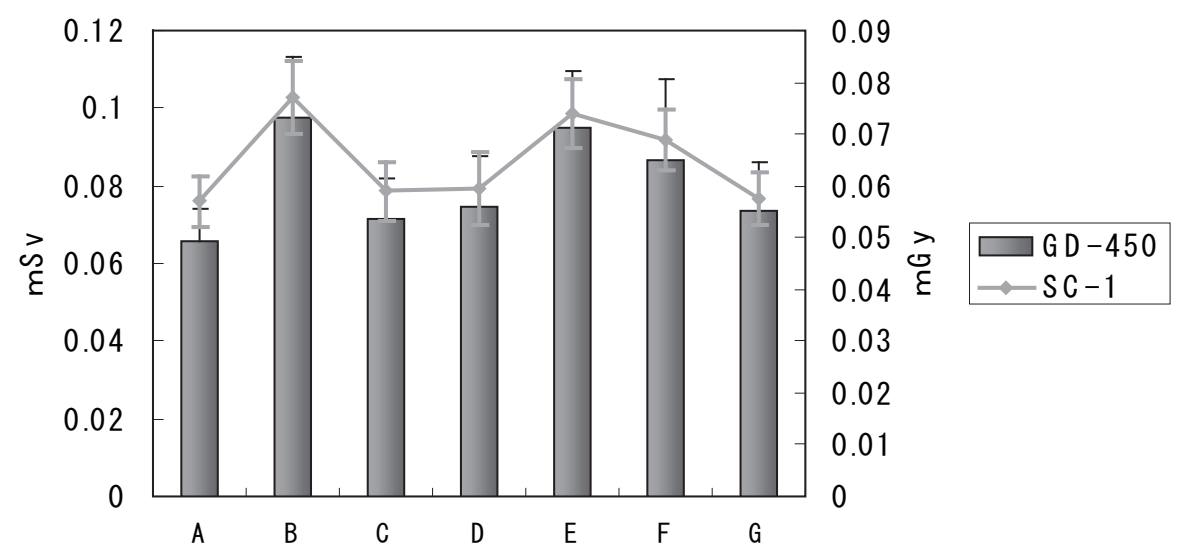

Fig. 7. Dose response at each point in Ishikawa prefecture (A: Tsurugi-machi, B: Tatsunokuchi, C: Inside of house of Mt. Shishiku, D: Outside of Mt. shishiku, E: Inside of Ogoya Mines, F: Outside of Ogoya Mines, G: Public health and Environmental Science) determined using GD-450 (shaded bars) or SC-1 (gray line) dosimeters. The units of GD-45 and SC-1 are mSv and mGy, respectively.

Previously, basic characteristic data obtained using GD were investigated by Asahi Techno Glass Co., Ltd. ${ }^{(1)}$ The relationship between radiation dose and dose response showed a very suitable linearity. This indicates that GD is an appropriate dosimeter for monitoring natural environmental background radiation at very low doses.

In addition, we evaluated the relationship between the dose obtained using the GD-450 dosimeter at each point and rainfall. However, we found that there was no relationship because the correlation coefficient was very low (data not shown). Therefore, we can use GD-450 as a suitable monitoring dosimeter for evaluating natural environmental background radiation dose without any effect of rainfall. It is a future work to evaluate the relationships between dose change tendencies per month measured using GD and other effects such as temperature and thunder on mountains.

\section{Conclusions}

The environmental natural background radiation doses at 7 points in Ishikawa prefecture, determined using the personal GD type GD-450, were compared with those determined using other dosimeters such as DIS based on a MOSFET with an ionization chamber and Luxel badge based on the OSL phenomenon in $\mathrm{Al}_{2} \mathrm{O}_{3}: \mathrm{C}$ phosphor. The actual doses were different from each other; however, the tendencies of each dose at each point were very similar. It can be said that the personal glass dosimeter, GD-450 will be very useful for monitoring not only personal dose but also natural background radiation dose. 


\section{Acknowledgements}

The authors wish to thank Mr. K.Yuda for his excellent assistance in the experiments. This work was partially supported by the foundation for Open-Research Center Program from the Ministry of Education, Culture, Sport, Science and Technology of Japan.

\section{References}

1 L. Duggan, M. Budzanowski, K. Przegietka, N. Reitsema, J. Wong and T. Kron: Radiat. Meas. 32 (2000) 335.

2 J. C. Saez-Vergara: Radiat. Protec. Dosim. 92 (2000) 83.

3 A. Sarai, N. Kuruta, K. Kamijo, N. Kubota, Y. Takei, H. Nanto, I. Kobayashi, H. Komori and K. Komura: J. Nucl. Sci. Technol, Suppl. 4 (2004) 474.

4 Y. Miyamoto, K. Kinoshita, S. Koyama, Y. Takei, H. Nanto, T. Kurobori, M. Sakakura, Y. Shimotsuma, K. Miura, K. Hirao and T. Yamamoto: Radioisotopes 58 (2009) 591 (in Japanese).

5 C. Wernli. Radiat. Protec. Dosim. 77 (1998) 253.

6 R. Yokota: OYO BUTURI 38 (1969) 1077 (in Japanese).

7 R. Kalchgruber and G. A. Wagner: Radiat. Meas. 41 (2006) 154.

8 X. Yang, H. Li, Q. Bi, Y. Cheng, Q. Tang, X. Qian and J. Xu: J. Lumin. 129 (2009) 566.

9 H. Fuchs, S. Stähler and M. Dittmar: Radiat. Protec. Dosim. 128 (2007) 120.

10 F. Vanhavere and P. Covens: Radiat. Protec. Dosim. Advance Access (2009) 274.

11 K. Nomura, T. Ikegami and N. Juto: Radioisotopes 51 (2002) 85. 